\title{
The menopausal symptoms and depressive symptoms were efficacy of interaction between levels and duration of exercise in perimenopausal women:a cross- sectional study
}

\section{Shuang-shuang Wei}

Hangzhou Maternity Hospital https://orcid.org/0000-0002-9457-9628

Zhi-fen Zhang ( $\nabla$ zhangzf@zju.edu.cn )

https://orcid.org/0000-0002-0025-8242

Wen-hua Liu

Hangzhou Maternity hospital

Shan-shan Tang

Hangzhou maternity Hospital

Jian Huang

Hangzhou Maternity Hospital

\section{Research article}

Keywords: Depressive symptoms, Exercise, Perimenopausal women, menopausal symptoms, menopause

Posted Date: March 6th, 2020

DOI: https://doi.org/10.21203/rs.3.rs-16257/v1

License: (c) (i) This work is licensed under a Creative Commons Attribution 4.0 International License.

Read Full License 


\section{Abstract}

Background Depressive disorder often adversely affects psychosocial and physical functioning. Exercise is one of the most commonly used alternatives for mood disorders and menopausal symptoms during the menopausal transition. This study aimed to investigate the interaction between levels and duration of exercise and their effects on menopausal symptoms and depressive symptoms.

Methods We enrolled 512 healthy women aged 40-60 years. Exercise history, menopausal symptoms (MKS), and depressive symptoms (HAMD) were determined based on self-report. Menopausal symptoms and depressive symptoms was analyzed using a 2-way analysis of variance(ANOVA) with levels of exercise and duration of exercise as factors, respectively. Significant main effects or interactions were examined using Fisher's Least Significant Difference (LSD) tests. Specific planned comparisons were made using Bonferroni corrections.

Results There were interactions between levels and duration of exercise on the change in menopausal symptoms and depressive symptoms, respectively $F(6,512)=3.597, F(6,512)=2.128, p<0.05]$. Pairwise comparisons of the interaction revealed that, with moderate duration of exercise, those women who did high level of exercise had lower scores of MKS and HAMD than those who seldom exercised $(P<0.05)$. Short or long duration of exercise and low or high levels of exercise was not effective at reducing scores of $\operatorname{MKS}$ and $\operatorname{HAMD}(p>0.05)$.

Conclusions The relationship between exercise and menopausal symptoms had a U-shaped trend. The relationship between exercise and depressive symptoms also had a U-shaped trend.

\section{Background}

Depressive disorder often adversely affects psychosocial and physical functioning, including poor occupational functioning, role functioning, social functioning, physical health, as well as leads to high levels of disability ${ }^{[1]}$. According to World Health Organization (WHO) reports, the major depressive disorder will be the second leading cause of incapacitating disease by $2020^{[2]}$. Although clinical relevance of this perceived increase in the prevalence of depressive disorder during menopausal transition has been questioned, the prevalence of depressive symptoms is particularly high during this period and may affect as many as one in every five women ${ }^{[3]}$. Therefore, it is important to gain a measure on how individuals cope with and overcome this symptoms.

One such therapy is exercise, which is one of the most commonly used alternatives for mood disorders and menopausal symptoms during the menopausal transition ${ }^{[4,5]}$. In a study conducted with 648 women, aged between 40 to 60 years, it was showed that, those who exercised regularly were less depressed and less symptomatic than women who did not exercise ${ }^{[5]}$. Another analysis of data involving 60 woman with symptoms of anxiety and depression during study period, the menopausal women could benefit from physical exercise, and attenuated the effects of psychological changes associated with menopause $\mathrm{e}^{[6]}$. 
A number of gaps exist in our knowledge about depressive symptoms and menopausal symptoms during the menopause transition. First, despite some studies suggested that exercise could alleviate menopausal symptoms and depressive symptoms, the studies carried out to examine more details of interaction between the levels and duration of exercise effect on depressive symptoms and menopausal symptoms was limited. Therefore, it remains unclear how the levels, and duration of exercise should be, and what combination is best ${ }^{[7,8,9]}$. Second, the duration of exercise are highly variable among studies that have investigated the effects of exercise on depressive symptoms and menopausal symptoms ${ }^{[4,8,10]}$. Finally, some studies typically employed high intensity exercise, which may itself be stressful, and this stress may, in turn, contributed to depressive and menopausal symptoms ${ }^{[4,8]}$. Therefore, the current study was focused on the depressive symptoms and menopausal symptoms regulated by different levels and duration of exercise among a sample of perimenopausal women in a clinical setting. This study provided an initial assessment of possible impact of different levels and duration of exercise on menopausal symptoms and depressive symptoms in perimenopausal women, and, further specifies, which combination between levels and duration of exercise is the best. This report also investigates whether high intensity exercise adversely affects depressive and menopausal symptoms.

\section{Methods}

\section{Study design\& settings}

In this cross-sectional study, all subjects were enrolled by means of purposive sampling from menopause outpatient clinics in the Hangzhou Maternity Hospital. The data collection was conducted from 1th January until 31th December 2019. In total, 1704 women were invited to participate in the study. Exercise history, menopausal symptoms (MKS), and depressive symptoms (HAMD) were determined based on self-report.

\section{Participants}

Inclusion criteria were age of $40-60$ years, $\mathrm{E}_{2}<30 \mathrm{pg} / \mathrm{L}, \mathrm{FSH}>40 \mathrm{IU} / \mathrm{L}$, and the ability to understand a questionnaire. Subjects with prior use of antidepressants, selective estrogen-receptor modulators or anxiolytics and diagnosed with major systemic illness were excluded in the screening stage. In total, 1704 women were invited to participate in the study and 958 perimenopausal women were selected to investigate the influence of exercise on perimenopause-associated symptoms. 201 subjects who had a history of hormone replacement therapy for the management of menopausal symptoms, 140 subjects with missing data on their HAMD questionnaire, 55 subjects with missing data on their MKS questionnaire, and 50 subjects with missing information on history of exercise were excluded from the analysis. Therefore, 512 women were finally eligible to be enrolled for this study. The study was approved by the ethics review board of the hospital. The study's objectives were explained in detail to each woman, and written informed consent was read and signed by all the participants.

\section{Measurements}


A cross-sectional study of menopausal symptoms and depressive symptoms was undertaken with female volunteers enrolled at menopause transition(The Early menopausal transition is defined as a persistent difference of 7 days or more in the length of consecutive cycles. The late menopausal transition is defined as the occurrence of amenorrhea of 60 days or longer. The early postmenopause is defined as FSH continues to increase and estradiol continues to decrease until approximately 2 years after the FMP ${ }^{[11]}$.). The questionnaire was used to collect the data and translated to local language(Chinese) by the head of the department of Obstetrics and Gynecology, Hangzhou Maternity Hospital The variables of the participant's details included current age, education, economy, alcohol ingestion, cigarette smoking, levels of exercise, duration of exercise, types of exercise, Modified Kupperman Indexs (MKS) and Hamilton Depression Scale(HAMD).

Women were required to report their exercise pattern in the previous year, including levels, duration and types of exercise. The levels of exercise was categorized as: (1) Seldom, (2) mild: 1-2times per month, (3) moderate: 1-2 times per week, (4) high: more than 3 times per week. The duration of exercise was categorized as: (1) short: 20-30 minutes per session, (2) moderate: 30-60 minutes per session, (3) long: more than 60 minutes per session. The types of exercise was categorized as: (1) brisk walking, (2) swimming, (3) dancing, (4) hiking.

The Modified Kupperman Indexs ${ }^{[12]}$ (MKS) is a 13-item self-report measure, including hot flushes/night sweats, paresthesia, dizziness, arthralgia/myalgia, headache, palpitations, formication, insomnia/sleep disturbance, depression, irritability, fatigue, urinary infection and sexual complaints. The total score ranges from 0 to 63 , calculated as the sum of all of the scores for each item. The total score were used to rate the degree of severity and divided into four categories: 0-6 (none), 7-15 (mild), 16-30 (moderate) and $>30$ (severe) .

Hamilton Depression Scale (HAMD) $)^{[13,14]}$ was used for measuring depressive symptoms. These scales have been used globally by various authors for measurement of depressive symptoms. The HAMD is a 24-item questionnaire to evaluate patients for depression-associated symptoms. Higher scores indicate a higher level of depressive symptoms and are divided into four categories: "Normal range" (score less than 8), "possibly depressive" (score 8-20), "mildly to moderately depressive" (score $>20$ ), and "markedly to severely depressive" (score >35). The HAMD can be classified into five categories of factor structure, including anxiety somatization, weight, cognitive disorders, block, sleep disorders, which not only reflect the specific characteristics of the patient's condition, but also the results of clinical symptoms of target group.

\section{Statistical analysis}

All statistical analyses were performed with SPSS 19.0. The baseline characteristics of the study participants were expressed as mean \pm standard deviation (SD), or number (\%). The relationships between menopause symptoms and depressive symptoms were assessed using Pearson's correlation analysis. Two-way repeated ANOVA was used to compare menopausal symptoms and depressive 
symptoms (factor $1=$ levels of exercise, factor $2=$ duration of exercise). Significant main effects or interactions were examined using Fisher's Least Significant Difference (LSD) tests. Specific planned comparisons were made using Bonferroni corrections. Differences were considered statistically significant at $P<0.05$ for all analyses.

\section{Results}

\section{The general characteristics of the Participants}

The age of the participants ranged from 40 to 60 years with a mean age of 48.23 ( $S D=4.69)$ years. The general characteristics of the study participants are shown in Table 1. Most menopause women were married (93.75\%), employed (75.78\%), had college or higher education $(67.38 \%)$, did not smoke (99.22\%), and did not consume alcohol (85.94\%).

\section{Menopausal symptoms versus depressive symptoms}

The mean score of menopausal symptoms and depressive symptoms in this sample were 9.53 $(S D=7.76), 5.75(S D=7.02)$ respectively. The none, mild, moderate and severe level of menopausal symptoms was $43.4 \%, 35.3 \%, 19.5 \%, 1.8 \%$ respectively. The normal, doubtful, mild to moderate, and marked level of depressive symptoms was $68.75 \%, 26.56 \%, 3.32 \%, 1.37 \%$ respectively.

The MKS was positively correlated with $\operatorname{HAMD}(r=0.63, p<0.001)$ using Pearson's correlations.

\section{Menopausal symptoms versus exercise}

Table 2 shows change in menopausal symptoms relative to different levels and duration of exercise in perimenopausal woman. Two-way ANOVA revealed main effects of duration of exercise $[F(2,512)=4.910$, $p<0.05]$ and the different levels of exercise $[F(3,512)=2.039, p=0.108]$. Moreover, there was also an interaction between levels and duration of exercise on changes in $\operatorname{MKS}[F(6,512)=3.597, p<0.05]$. Pairwise comparisons of the interaction revealed that, with seldom exercise, those women who did long duration of exercise had lower menopausal symptoms scores than those who mild duration of exercised $(P<0.05)$. With moderate duration of exercise, those women who did high level of exercise had lower menopausal symptoms scores than those who seldom exercised $(P<0.05)$.

\section{Depressive symptoms versus exercise}

Table 3 shows change in depressive symptoms relative to different levels and duration of exercise in perimenopausal woman. Two-way ANOVA revealed main effects of duration of exercise $[F(2,512)=4.661$, $p<0.05]$ and different levels of exercise $[F(3,512)=1.064, p=0.364]$. Moreover, there was also a significant interaction between levels and duration of exercise on changes in $\operatorname{HAMD}[F(6,512)=2.128, p<0.05]$.

Pairwise comparisons of the interaction revealed that, with mild level of exercise, those women who did long duration of exercise had lower depressive symptoms scores than those who moderate duration of 
exercised $(P<0.05)$. With moderate duration of exercise, those women who did high level of exercise had lower depressive symptoms scores than those who seldom exercised $(P<0.05)$.

\section{Discussion}

Health promotion for perimenopausal women is gaining considerable attention due to the various health care issues raised with the advent of an aging society. Women in this age group are not only bothered by menopausal symptoms but are also at increased risk for mood disorders ${ }^{[15]}$. Some researches had identified perimenopause as a period of increased risk for both first-episode depression and for depression reoccurrence ${ }^{[16]}$, and a positive correlation between the menopausal symptoms and depressive symptoms ${ }^{[17,18]}$. In the present study, we found that $31.75 \%, 56.6 \%$ of menopause women were affected by the symptom of depressed mood and menopausal symptoms respectively, which was consistent with several studies conducted in other countries, such as Spain, Japan, USA and México ${ }^{[19,20]}$. We found a positive correlation of menopausal symptoms with depressive symptoms, which provided effective evidence suggesting that women who experienced more severe menopausal symptoms are more likely to exhibit increased levels of depressive symptoms as they move through the menopausal transition ${ }^{[17,18,20]}$. Reason for depressive symptoms during menopausal period may be due to alteration in the levels of reproductive hormones which may directly affect central neurotransmitter activity and contribute to a dysregulation of the hypothalamicpituitaryadrenal axis, leading to onset of depressive symptoms in vulnerable women ${ }^{[21,22,23]}$. Another reason is that menopausal period could improve the levels of pro-inflammatory cytokines, such as IL-6, IL-8, TNF-a, which are associated with the expression of behavioral characteristic of decreased psychological well-being ${ }^{[24,25]}$. Moreover, Women may meet great changes and experienced many negative events in their social lives during menopausal transition such as financial hardship, stressful life events, poor social support, empty nest and gap generation, which brings stress to them and contributes to develop major depressive disorder or depressive symptoms ${ }^{[26]}$. Finally, Other observational studies have found that past depression was a robust predictor of depression during menopausal transition and an early menopause may increase the risk of depressive symptoms, as may the presence of persistent vasomotor symptoms ${ }^{[3]}$. However, a cross-sectional and longitudinal population-based studies concluded that although depressive symptoms were more frequent during menopausal transition, depressive disorders were not ${ }^{[27]}$, which need further research.

Previous studies had shown a positive association between physical exercise and the promotion of physical, mental and social health, thus enhanced quality of life during this stage of life ${ }^{[6,16]}$. Regular physical exercise during the menopausal age have shown to alleviate the menopausal symptoms as reported in some studies with women who exercise regularly tend to have fewer symptoms. But the evidence for the effectiveness of exercise interventions for menopausal symptoms is still unclear, with some studies findings benefits and others not ${ }^{[5,21,28-31]}$. It is possible that bouts of strenuous exercise might trigger hot flushes ${ }^{[4,32]}$. Our study indicated that the relationship between exercise and menopausal symptoms had a U-shaped trend. Although the relationship between short duration of exercise and 
menopausal symptoms having clinical significance is questionable, it is estimated that from seldom to high level of exercise would result in 0.6 times decrease in MS when the duration of exercise is short. In this regard, short duration of exercise may relieve menopausal symptoms by increasing levels of exercise. Moreover, the decreased MKS by increasing levels of exercise, experienced only in moderate duration of exercise condition might suggest that there is beneficial effect of exercise on menopause ${ }^{[33]}$, which needs to be further confirmed by prospective studies. For example, flexibility in exercise, such as 20-60 minutes each day yoga, could reduced menopause symptoms ${ }^{[31]}$. However, one randomized controlled trial study suggested that just 10 minutes of mild exercise before bedtime decreased menopausal symptoms during their menopausal transition, and they focused on the effects of light-intensity exercise, specifically stretching, on menopausal symptoms ${ }^{[34]}$. These contrasting results maybe explained by differences in the type of exercise, which are categorized into four broad areas of physical fitness: aerobic exercise, muscle strengthening, flexibility, and balance ${ }^{[23]}$. In our study, we almost used aerobic exercise, which involves the use of large muscle groups, including brisk walking, swimming, dancing and hiking. In addition, our data showed that high level and long duration of exercise usually practiced by those with incident menopausal disorders ${ }^{[9]}$ did not seem to be effective in lowering menopausal symptoms. These negative findings have important clinical implication. The effect of exercise was not dose dependent, since the high level and long duration of exercise group experienced more symptoms than the moderate level and duration of exercise group ${ }^{[9]}$. Hence, on the one hand, with short or moderate duration of exercise, those women could relieve menopausal symptoms by increasing levels of exercise. On the other hand, with lower moderate levels of exercise, those women could relieve menopausal symptoms by increasing duration of exercise. We also strongly didn't recommend high level and long duration of exercise for perimenopausal women to alleviate their menopausal symptoms.

This study demonstrated that low levels and short duration of exercise had no significant effect on depressive symptoms in line with previous research on perimenopausal women ${ }^{[7,35]}$. This is inconsistent with the finding that, 10 minutes of light-intensity exercise before bedtime decreased depressive symptoms in middle-aged women ${ }^{[34]}$. These contrasting results maybe explained by differences in the etiology of depressive symptoms and intensity of exercise, which may be interacting with one another. Moreover, the decreased HAMD by increasing level of exercise experienced in moderate duration of exercise condition, might suggest that there is beneficial effect of exercise on depressive symptoms ${ }^{[1]}$. Is it plausible that different mechanism are at work with respect to the moderate and short duration of exercise? Several studies have demonstrated that oxidative stress and pro-inflammatory cytokines, such as IL-1 $\beta$, IL-6, NLRP3,8-hydroxy-2'-deoxy -guanosine, which were increased during menopause contributes to development of depression like behavior ${ }^{[13,36-38]}$. However, exercise could decrease this proinflammatory cytokines and oxidative stress and consequently ameliorate depressive symptoms $^{[13,15,16,39]}$, whereas the short duration of exercise may not be adequate for such a change. Furthermore, Schuch et al ${ }^{[40]}$ showed that moderate intensity water-base exercise appeared to be an efficient strategy to improve the quality of life in women by their anti-depressing effect. Therefore, regarding depressive symptoms, we suggest that moderate duration exercise is ideal. Another interesting 
finding is the lack of difference in depressive symptom when exercise level and/or duration were high. Although one would expect that people suffering from depressive symptoms would be ameliorated by high intensity exercise ${ }^{[7,35]}$, the data does not support this assumption. The possible explanation concerned with this phenomenon could be, post-exercise depression (PED) that persists for 20-30times after exhaustive exercise due to muscle fatigue ${ }^{[41]}$. PED may be caused by intracortical mechanisms, because of not changing spinal reflexes, peripheral and subcortical components ${ }^{[31,42]}$. Another potential explanation for PED include long-term depression, decreased excitability of intracortical glutamatergic networks, and increased excitability of inhibitory GABAergic networks ${ }^{[41,43]}$. However, due to paucity of studies and methodological limitations, conclusions regarding the underlying mechanisms for the antidepressant effect of high intensity exercise in depressive symptoms are not currently possible.

To our knowledge, this is one among the few studies which investigated the relationships between depressive symptoms and menopausal symptoms in perimenopausal women, and explored the duration and levels of exercise as to achieve the best dose-response. However, there are some study limitations. First, the cross-sectional study design means that a causal relationship cannot be identified; thus a longitudinal study is required to achieve higher levels of evidence regarding a causal relationship. Second, there may have been some information bias in terms of menopausal symptoms and depressive symptoms because these variables were measured using self-reported questionnaires. Studies of depression in perimenopausal women have generally relied on the assessment of depressive symptoms rather than a formal diagnosis of depression, and therefore, it is possible that misclassification could have occurred. Moreover, exercise measurement relies on memory of exercise, over one year memory could be a problem and levels of exercise may vary over this period. Lastly, although physical exercise is widely promoted as a nonpharmacological intervention for menopausal symptoms and depressive symptoms prevention, not all types of exercise appear to be useful in reducing the risk of menopausal symptoms and depression in woman. Our study in perimenopausal women have not explored the type of exercise, which needs further research.

\section{Conclusions}

In conclusion, there was a significant positive correlation between the severity of menopausal symptoms and depressive symptoms. With short or moderate duration of exercise, those women could relieve menopausal symptoms and depressive symptoms by increasing levels of exercise. With lower moderate levels of exercise, those women could relieve menopausal symptoms and depressive symptoms by increasing duration of exercise. High levels and long duration of exercise did not seem to be effective in lowering menopausal and depressive symptoms.

\section{Abbreviations}

MKS

Modified Kupperman Indexs 
HAMD

Hamilton Depression Scale

\section{Declarations}

\section{Ethics approval and consent to participate}

The Ethical approval for this research was obtained from the Institutional Review Committee of Hangzhou Maternity Hospital. All study participants provided a written informed consent to agree the data to be used in national statistics and research.

\section{Consent to publish}

Not applicable.

\section{Availability of data and materials}

All data generated or analysed during this study are included in this published article.

\section{Competing interests}

The authors declare that they have no competing interests.

\section{Funding}

This research was supported by Scientific research foundation of national health and Health Commission (grant number:WKJ-ZJ-2010). The funders had no role in study design, data collection and analysis, decision to publish, or preparation of the manuscript.

\section{Authors' Contributions}

SS Wei has contributed in designing, collecting data, analyzing data, writing and revising drafts of this manuscript. ZF Zhang has contributed in designing the study, conceptualisation, analysing and interpretation of data, and writing the revised draft of the manuscript. WH Liu and SS Tang has contributed in analyzing data. J Huang has contributed in writing the revised draft of the manuscript.

\section{Acknowledgements}

Not applicable.

\section{References}

[1]. Klil-Drori S, Klil-Drori AJ, Pira S, Rej S. Exercise Intervention for Late-Life Depression: A Meta-Analysis. J Clin Psychiatry. 2020 Jan 21;81(1) 
[2].Silveira H, Moraes H, Oliveira N, Coutinho ES, Laks J, Deslandes A. Physical Exercise and Clinically Depressed Patients: A Systematic Review and Meta-Analysis. Neuropsychobiology 2013;67(2):61-8.

[3]. Almeida OP, Marsh K, Flicker L, Hickey M, Sim M, Ford A. Depressive symptoms in midlife: the role of reproductive stage. Menopause 2016;23(6):669-75.

[4].Kim MJ, Cho J, Ahn Y, Yim G, Park HY. Association between physical activity and menopausal symptoms in perimenopausal women. BMC Womens Health 2014:3(14):122.

[5].Lee Y, Kim H. Relationships between menopausal symptoms, depression, and exercise in middle-aged women: A cross-sectional survey. International Journal of Nursing Studies 2008,45:1816-1822.

[6].Villaverde Gutiérrez C, Torres Luque G, Ábalos Medina GM. Influence of exercise on mood in postmenopausal women. J Clin Nurs 2012 Apr;21(7-8):923-8.

[7].Balchin R, Linde J, Blackhurst D, Rauch HL, Schönbächler G. Sweating away depression? The impact of intensive exercise on depression. J Affect Disord 2016;200:218-21.

[8].Grindler NM, Santoro NF. Menopause and exercise. Menopause 2015 ;22(12):

1351-8.

[9]. Ibrahim ZM, Sayed Ahmed WA, El-Hamid SA. Prevalence of menopausal related symptoms and their impact on quality of life among Egyptian women. Clin Exp Obstet Gynecol. 2015;42(2):161-7.

[10].Schuch FB, Vasconcelos-Moreno MP, Borowsky C, Zimmermann AB, Rocha NS, Fleck MP. Exercise and severe major depression: Effect on symptom severity and quality of life at discharge in an inpatient cohort. J Psychiatr Res 2015;61:25-32.

[11]. Harlow SD, Gass M, Hall JE, Lobo R, Maki P, Rebar RW, et al. Executive summary of the Stages of Reproductive Aging Workshop + 10: addressing the unfinished agenda of staging reproductive aging. Menopause 2012;19(4):387-95.

[12]. Tao M, Shao H, Li C, Teng Y. Correlation between the modified Kupperman Index and the Menopause Rating Scale in Chinese women. Patient Prefer Adherence. 2013;7:223-9

[13]. Baek SE, Lee GJ, Rhee CK, Rho DY, Kim DH, Huh S3, et al. Decreased Total Antioxidant Activity in Major Depressive Disorder Patients Non-Responsive to Antidepressant Treatment. Psychiatry Investig 2016;13(2):222-6.

[14].Lee BH, Park YM. How Childhood Maltreatment Is Related to Suicidality, Bipolarity and Central Serotonergic Activity in Patients with Major Depressive Disorder: A Cross-Sectional Pilot Study. Psychiatry Investig 2016;13(2):190-5. 
[15]. Fu JX, Luo Y, Chen MZ, Zhou YH, Meng YT, Wang T, et al. Associations among menopausal status, menopausal symptoms, and depressive symptoms in midlife women in Hunan Province, China. Climacteric. 2020 Jan 17:1-8

[16].Wang Y, Xu Y, Sheng H, Ni X, Lu J. Exercise amelioration of depression-like behavior in OVX mice is associated with suppression of NLRP3 inflammasome activation in hippocampus. Behav Brain Res 2016 1;307:18-24.

[17]. Humeniuk E, Bojar I, Gujski M, Raczkiewicz D. Effect of symptoms of climacteric syndrome, depression and insomnia on self-rated work ability in peri- and post-menopausal women in non-manual employment. Ann Agric Environ Med. 2019 Dec 19;26(4):600-605.

[18].Borkoles E, Reynolds N, Thompson DR, Ski CF, Stojanovska L, Polman RC. The role of depressive symptomatology in peri- and post-menopause. Maturitas 2015;81(2):306-10 .

[19].Wang HL, Booth-LaForce C, Tang SM, Wu WR, Chen CH. Depressive symptoms in Taiwanese women during the peri- and post-menopause years: associations with demographic, health, and psychosocial characteristics. Maturitas $2013 ; 75(4): 355-60$.

[20]. Pérez-López FR1, Pérez-Roncero G, Fernández-Iñarrea J, Fernández-Alonso AM, Chedraui P, Llaneza P, et al. Resilience, depressed mood, and menopausal symptoms in postmenopausal women. Menopause 2014;21(2):159-64

[21].Soares CN. Depression in Peri- and Postmenopausal Women: Prevalence, Pathophysiology and Pharmacological Management. Drugs Aging 2013;30(9):677-85

[22].Bansal P, Chaudhary A, Soni RK, Sharma S, Gupta VK, Kaushal P. Depression and anxiety among middle-aged women: A community-based study. J Family Med Prim Care 2015;4(4):576-81.

[23].Gibbs Z, Lee S, Kulkarni J. What factors determine whether a woman becomes depressed during the perimenopause? Arch Womens Ment Health 2012 ;15(5)囚323-32.

[24].Maldonado-Bouchard S, Peters K, Woller SA. Inflammation is increased with anxiety- and depressionlike signs in a rat model of spinal cord injury. Brain Behav Immun 2016;51:176-95.

[25].Ghosh M, Rodriguez-Garcia M, Wira CR. The Immune System in Menopause: Pros and Cons of Hormone Therapy. J Steroid Biochem Mol Biol 2014;142:171-5.

[26].Li Y, Yu Q, Ma L, Sun Z, Yang X. Prevalence of depression and anxiety symptoms and their influence factors during menopausal transition and postmenopause in Beijing city. Maturitas. 2008 Nov 20;61(3):238-42.

[27].Cohen LS, Soares CN, Vitonis AF, Otto MW, Harlow BL. Risk for new onset of depression during the menopausal transition: the Harvard study of moods and cycles. Arch Gen Psychiatry 2006;63(4):385-90. 
[28]. Juang KD, Wang SJ, Lu SR, Lee SJ, Fuh JL. Hot flashes are associated with psychological symptoms of anxiety and depression in peri- and post- but not premenopausal women. Maturitas. 2005 Oct 16;52(2):119-26.

[29].Du J, Zhang D, Yin Y. The Personality and Psychological Stress Predict Major Adverse Cardiovascular Events in Patients With Coronary Heart Disease After Percutaneous Coronary Intervention for Five Years. Medicine (Baltimore) 2016;95(15):e3364

[30]. Luoto R, Moilanen J, Heinonen R, Mikkola T, Raitanen J, Tomas E, et al. Effect of aerobic training on hot flushes and quality of life-a randomized controlled trial. Ann Med 2012;44(6):616-26.

[31].Newton KM, Reed SD, Guthrie KA, Sherman KJ, Booth-LaForce C, Caan B, et al. Efficacy of Yoga for Vasomotor Symptoms: A Randomized Controlled Trial. Menopause 2014;21(4):339-46.

[32].Stefanopoulou E, Shah D, Shah R, Gupta P, Sturdee DW, Hunter MS. The International Menopause Study of Climate, Altitude, Temperature (IMS-CAT) and vasomotor symptoms. Climacteric 2014;17(4):417-24

[33]. Mansikkamäki K, Nygård CH, Raitanen J, Kukkonen-Harjula K, Tomás E , Reetta Rutanen R, et al. Hot flushes among aging women: A 4-year follow-up study to a randomised controlled exercise trial. Maturitas 2016;88:84-9.

[34].Kai Y, Nagamatsu T, Kitabatake Y, Sensui H. Effects of stretching on menopausal and depressive symptoms in middle-aged women: a randomized controlled trial. Menopause 2016;23(8):827-32.

[35].WangHL, Booth-LaForce C, Tang SM, Wu WR, Chen CH. Depressive symptoms in Taiwanese women during the peri- and post-menopause years: Associations with demographic, health, and psychosocial characteristics. Maturitas $2013 ; 75(4): 355-60$

[36].Tartibian B, FitzGerald LZ, Azadpour N, Maleki BH. A randomized controlled study examining the effect of exercise on inflammatory cytokine levels in postmeno

-pausal Women. Post Reprod Health 2015;21(1):9-15.

[37].Xu Y, Sheng H, Bao Q, Wang Y, Lu J, Ni X. NLRP3 inflammasome activation mediates estrogen deficiency-induced depression- and anxiety-like behavior and hippocampal inflammation in mice. Brain Behav Immun 2016;56:175-86.

[38].Hallgren M , Nakitanda OA, Ekblom Ö, Herring MP , Owen N, Dunstan D , et al. Habitual physical activity levels predict treatment outcomes in depressed adults: A prospective cohort study. Prev Med 2016;88:53-8.

[39].Rethorst CD, Toups MS, Greer TL, Nakonezny PA, Carmody TJ, Grannemann BD et al. Proinflammatory cytokines as predict -ors of antidepressant effects of exercise in major depressive disorder. 
Mol Psychiatry 2013;18(10):1119-24.

[40].Schuch FB, Pinto SS, Bagatini NC, Zaffari P, Alberton CL, Cadore EL, et al. Water-Based Exercise and Quality of Life in Women: The Role of Depressive Symptoms. Women Health 2014;54(2):161-75

[41]. Miyaguchi S, Kojima S, Kirimoto H, Tamaki H, Onishi H. Do Differences in Levels, Types, and Duration of Muscle Contraction Have an Effect on the Degree of Post-exercise Depression? Front Hum Neurosci. 2016 Apr 29;10:159.

[42]. Bridoux A, Créange A, Sangare A, Ayache SS, Hosseini H, Drouot X, et al. Impaired sleep-associated modulation of post-exercise corticomotor depression in multiple sclerosis. J Neurol Sci. 2015 Jul 15;354(1-2):91-6.

[43].Teo WP, Rodrigues JP, Mastaglia FL, Thickbroom GW. Changes in corticomotor excitability and inhibition after exercise are influenced by hand dominance and motor demand. Neuroscience 2012;17;210:110-7.

\section{Tables}

Table 1 Characteristics of the study population

\begin{tabular}{ccc}
\hline & Number of cases & Percentage (\%) \\
\hline Age (years) & 291 & 56.84 \\
$40-50$ & 221 & 43.16 \\
$50-60$ & 480 & 93.75 \\
Marital status & 2 & 0.39 \\
Married & 1 & 0.20 \\
Single & 4 & 0.78 \\
Separated & 25 & 4.88 \\
Widowed & 0 & 0 \\
Divorced & & 12.89 \\
Other & 66 & 19.73 \\
Educational background & 101 & 66.01 \\
Elementary school & 338 & 1.37 \\
Middle school & 7 & 75.78 \\
College & 388 & 24.22 \\
Other & 124 & 99.22 \\
Employment status & & 0.78 \\
Unemployed & 508 & 85.94 \\
Employed & 4 & 14.06 \\
Smoking status & & \\
No smoking & 440 & \\
Smoking & 72 & \\
Alcohol consumption & & \\
Yo & & \\
Yes & & \\
\hline
\end{tabular}


Table 2 MKS (mean \pm standard error) at different duration and levels of exercise and a two-way repeated measure ANOVA from differences duration and levels of exercise.

\begin{tabular}{cccc}
\hline & \multicolumn{3}{c}{$\begin{array}{c}\text { MKS (mean } \pm \text { standard error) } \\
\text { Levels of exercise }\end{array}$} \\
\hline & short & moderate & long \\
Seldom & $10.32 \pm 7.97 \triangle$ & $19.30 \pm 8.21^{*} \triangle$ & $4.00 \pm 0.10 \triangle$ \\
mild & $13.50 \pm 5.04$ & $11.42 \pm 8.22^{*}$ & $6.29 \pm 5.25$ \\
moderate & $7.83 \pm 12.05$ & $8.72 \pm 7.36^{*}$ & $10.86 \pm 6.54$ \\
high & $4.00 \pm 4.10$ & $8.10 \pm 6.88^{*}$ & $8.32 \pm 7.20$ \\
\hline Two-way repeated measures ANOVA & $F$-value & $P$-value \\
\hline Levels of exercise & 2.039 & 0.108 \\
Duration of exercise & 4.910 & 0.008 \\
Levels of exercise $\times$ Duration of exercise & 3.597 & 0.002 \\
\hline
\end{tabular}

$*=$ statistically significant between different levels of exercise $(p<0.05)$.

$\triangle$ = statistically significant between different duration of exercise $(p<0.05)$.

Table 3 HAMD (mean \pm standard error)at different duration and levels of exercise and a two-way repeated measures ANOVA from different duration and levels of exercise.

\begin{tabular}{|c|c|c|c|}
\hline \multicolumn{4}{|c|}{ HAMD (mean \pm standard error) } \\
\hline \multirow[t]{2}{*}{ Levels of exercise } & \multicolumn{3}{|c|}{ Duration of exercise } \\
\hline & short & moderate & long \\
\hline Seldom & $6.47 \pm 7.56$ & $11.90 \pm 10.79^{*}$ & $1.00 \pm 0.10$ \\
\hline mild & $7.00 \pm 4.96$ & $7.36 \pm 7.34^{*} \triangle$ & $2.00 \pm 2.15^{\triangle}$ \\
\hline moderate & $5.00 \pm 6.47$ & $5.46 \pm 7.17^{*}$ & $5.93 \pm 6.63$ \\
\hline high & $0.67 \pm 1.03$ & $4.97 \pm 6.73^{*}$ & $5.02 \pm 6.25$ \\
\hline \multicolumn{2}{|c|}{ Two-way repeated measures ANOVA } & $F$-value & $P$-value \\
\hline \multicolumn{2}{|c|}{ Levels of exercise } & 1.064 & 0.364 \\
\hline \multicolumn{2}{|c|}{ Duration of exercise } & 4.661 & 0.010 \\
\hline \multicolumn{2}{|c|}{ Levels of exercisex Duration of exercise } & 2.128 & 0.049 \\
\hline
\end{tabular}

a

$*=$ statistically significant between different levels of exercise $(p<0.05)$.

$\triangle$ = statistically significant between different duration of exercise $(p<0.05)$.

Figures 


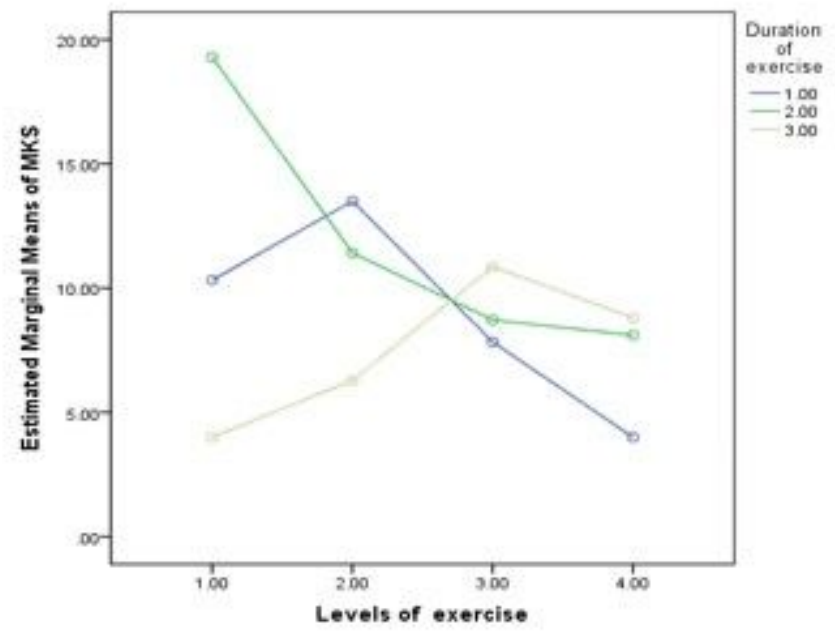

Figure 1

The interaction betweenlevels and duration of exercise on MKS

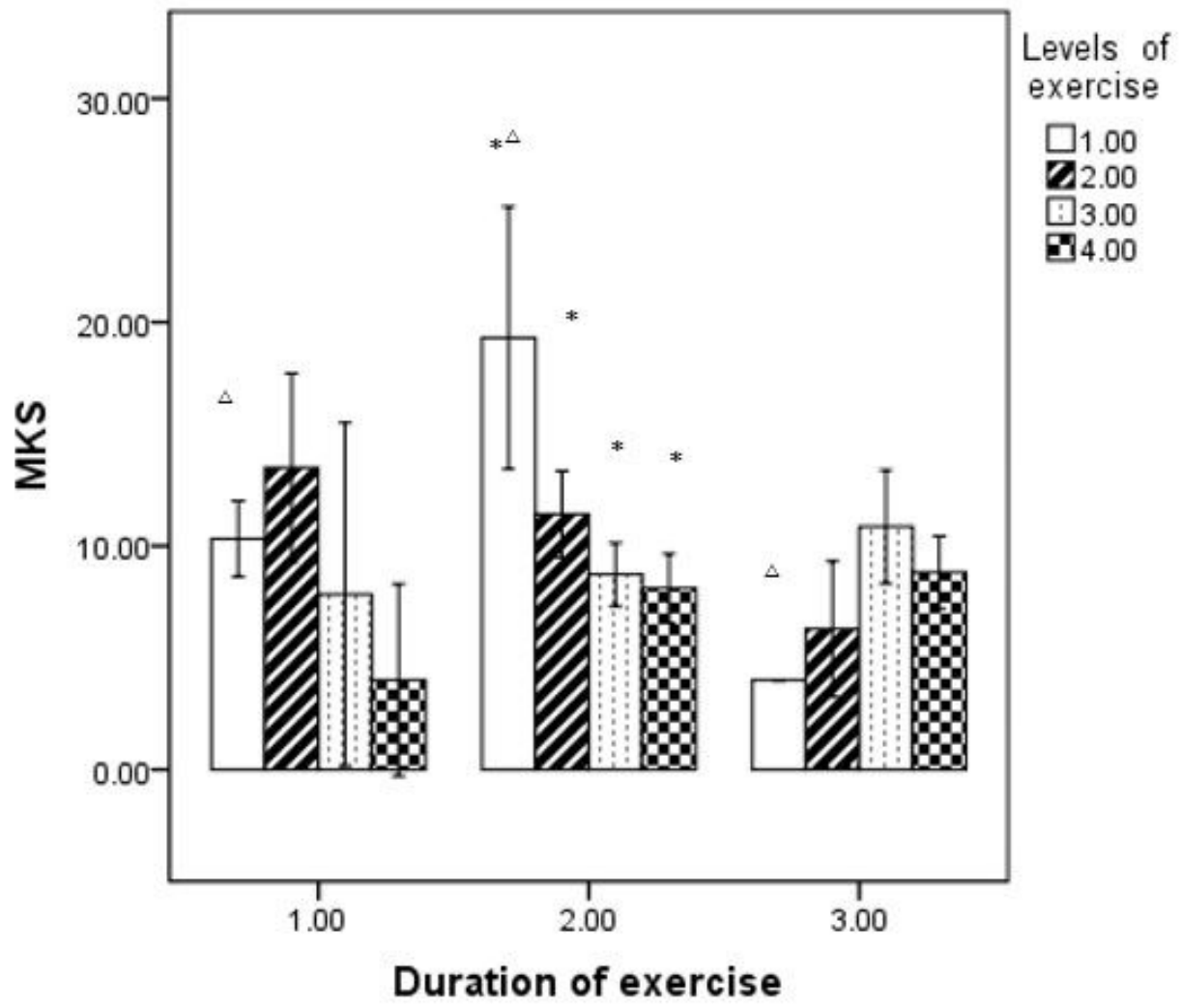

Figure 2 
effect of different levels and duration of exercise on menopausal symptoms Levels of exercise $₫ 1$. Seldom, 2.mild: 1-2 times per month, 3. moderate: 1-2 times per week, 4. high:more than 3 times per week. Duration of exercise: 1.short: 20-30 minutes per session, 2. moderate:30-60 minutes per session, 3 . long:more than 60 minutes per session. * = statistically significantbetweendifferent levels of exercise $(p<$ $0.05) . \triangle=$ statistically significant betweendifferent duration of exercise $(p<0.05)$.

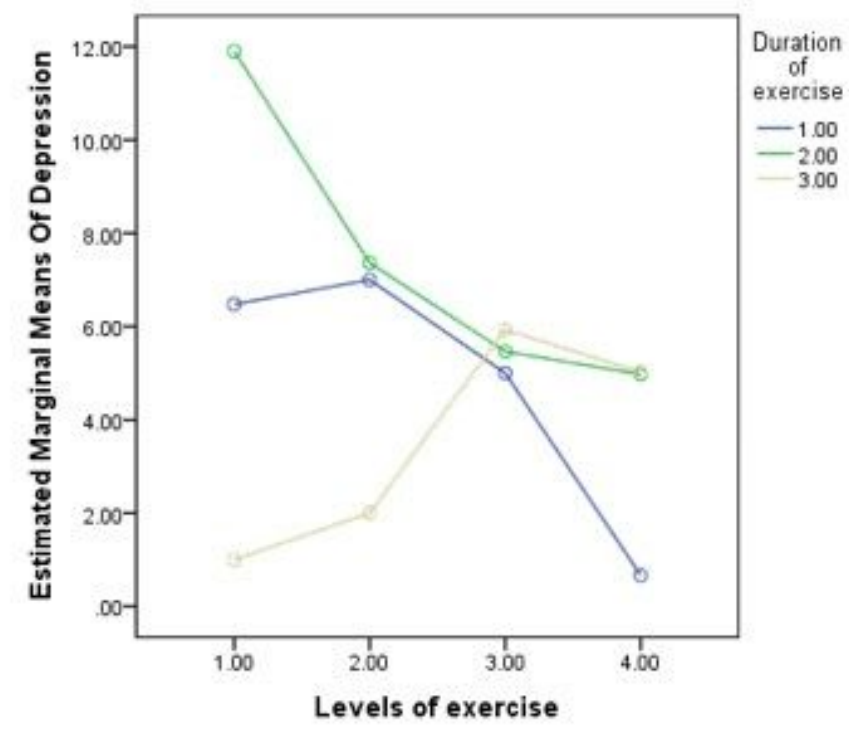

\section{Figure 3}

The interaction between levels and duration of exercise on HAMD. Levels of exercise冈1. Seldom, 2.mild: 12 times per month, 3. moderate: 1-2 times per week, 4. high:more than 3 times per week. Duration of exercise: 1.short: 20-30 minutes per session, 2. moderate:30-60 minutes per session, 3. long:more than 60 minutes per session. 


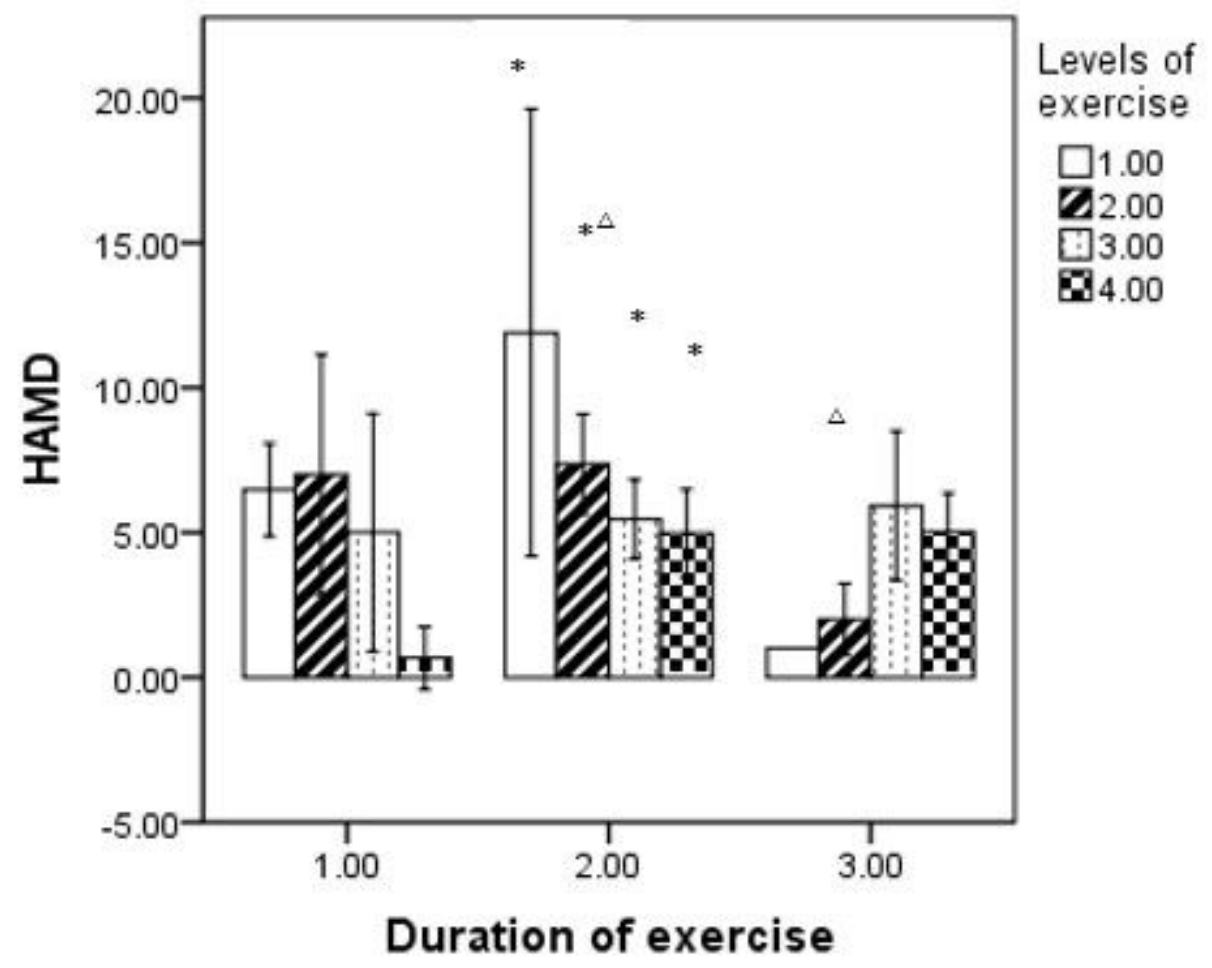

Figure 4

Effect of different levels and duration of exercise on depressive symptoms Levels of exercise $₫ 1$. Seldom, 2.mild: 1-2 times per month, 3. moderate: 1-2 times per week, 4. high:more than 3 times per week.

Duration of exercise: 1.short: 20-30 minutes per session, 2. moderate:30-60 minutes per session, 3 . long:more than 60 minutes per session. * = statistically significant between different levels of exercise $(p$ $<0.05) . \Delta=$ statistically significant betweendifferent duration of exercise $(p<0.05)$.

\section{Supplementary Files}

This is a list of supplementary files associated with this preprint. Click to download.

- STROBEchecklistv4combined.doc 